\title{
Impact of aggregation headers on aggregating small MSDUs in 802.11n WLANs
}

\begin{abstract}
The main goal of the IEEE $802.11 \mathrm{n}$ standard is to achieve more than $100 \mathrm{Mbps}$ of throughput at the MAC service access point. This high throughput has been achieved via many enhancements in both the physical and MAC layers. A key enhancement is frame aggregation which reduces the overheads and increases the channel utilization efficiency. The MAC layer defines A-MSDU and A-MPDU frame aggregations in which MAC overheads are squeezed by aggregating multiple frames into a single large frame before being transmitted. As a consequence of the aggregation, new aggregation headers are introduced and become parts of the transmitted frame. The existence of such headers will have a negative impact on the performance, especially when aggregating frames of small payloads. In this paper, we have analysed the aggregation headers of the 802.11n aggregation schemes and introduced an MSDU frame aggregation that reduces the header's overhead and supports the applications that have a small frame size such as VoIP.
\end{abstract}

Keyword: Frame aggregation; Block ACK; A-MSDU; Next generation networks; 802.11n 